\title{
BMJ
}

\section{Long term treatment with metformin in patients with type 2 diabetes and risk of vitamin B-12 deficiency: randomised placebo controlled trial}

\author{
Jolien de Jager, resident, ${ }^{1,2}$ Adriaan Kooy, internist, ${ }^{2,3}$ Philippe Lehert, professor of statistics, ${ }^{4}$ Michiel G \\ Wulffelé, general practitioner, ${ }^{2,3}$ Jan van der Kolk, biochemical engineer, ${ }^{5}$ Daniël Bets, program manager, ${ }^{6}$ Joop \\ Verburg, chief laboratory attendant, ${ }^{5}$ Ab J M Donker, professor of internal medicine, ${ }^{7}$ Coen D A Stehouwer, \\ professor and chair ${ }^{8}$
}

Department of Ophthalmology,

Academic Medical Center

Amsterdam, Netherlands

${ }^{2}$ Bethesda Diabetes Research Centre, Bethesda General

Hospital, Hoogeveen, Netherlands

${ }^{3}$ Department of Internal Medicine, Bethesda General Hospital, Hoogeveen, Netherlands

${ }^{4}$ Department of Statistics, Faculty of Economics, Facultés

Universitaires Catholiques de

Mons, Louvain Academy, Mons,

Belgium

${ }^{5}$ Clinical Laboratory, Bethesda General Hospital, Hoogeveen, Netherlands

${ }^{6}$ Clinical Research and Development, Merck Netherlands, Amsterdam, Netherlands

${ }^{7}$ Department of Internal Medicine, Free University Medical Center. Amsterdam, Netherlands

${ }^{8}$ Department of Internal Medicine, Maastricht University Medical Centre, Maastricht, Netherlands

Correspondence to: CD A

Stehouwer

cda.stehouwer@mumc.nl

Cite this as: BMJ 2010;340:c218 doi:10.1136/bmj.c2181

\section{ABSTRACT}

Objectives To study the effects of metformin on the incidence of vitamin B-12 deficiency ( $(150 \mathrm{pmol} / \mathrm{l})$, low concentrations of vitamin B-12 (150-220 pmol/l), and folate and homocysteine concentrations in patients with type 2 diabetes receiving treatment with insulin.

Design Multicentre randomised placebo controlled trial. Setting Outpatient clinics of three non-academic hospitals in the Netherlands.

Participants 390 patients with type 2 diabetes receiving treatment with insulin.

Intervention $850 \mathrm{mg}$ metformin or placebo three times a day for 4.3 years.

Main outcome measures Percentage change in vitamin B12 , folate, and homocysteine concentrations from baseline at4, 17, 30, 43, and 52 months.

Results Compared with placebo, metformin treatment was associated with a mean decrease in vitamin B-12 concentration of $-19 \%$ (95\% confidence interval $-24 \%$ to $-14 \%$; $\mathrm{P}<0.001)$ and in folate concentration of $-5 \%(95 \%$ $\mathrm{Cl}-10 \%$ to $-0.4 \% ; \mathrm{P}=0.033)$, and an increase in homocysteine concentration of $5 \%(95 \% \mathrm{Cl}-1 \%$ to $11 \%$; $\mathrm{P}=0.091)$. After adjustment for body mass index and smoking, no significant effect of metformin on folate concentrations was found. The absolute risk of vitamin B-12 deficiency ( $(150 \mathrm{pmol} / \mathrm{l})$ at study end was 7.2 percentage points higher in the metformin group than in the placebo group $(95 \% \mathrm{Cl} 2.3$ to $12.1 ; \mathrm{P}=0.004)$, with a number needed to harm of 13.8 per 4.3 years $(95 \% \mathrm{Cl}$ 43.5 to 8.3). The absolute risk of low vitamin B-12 concentration (150-220 pmol/l) at study end was 11.2 percentage points higher in the metformin group $(95 \% \mathrm{Cl}$ 4.6 to $17.9 ; \mathrm{P}=0.001)$, with a number needed to harm of 8.9 per 4.3 years $(95 \% \mathrm{Cl} 21.7$ to 5.6$)$. Patients with vitamin B-12 deficiency at study end had a mean homocysteine level of $23.7 \mu \mathrm{mol} / \mathrm{l}(95 \% \mathrm{Cl} 18.8$ to 30.0 $\mu \mathrm{mol} / \mathrm{l})$, compared with a mean homocysteine level of $18.1 \mu \mathrm{mol} / \mathrm{l}(95 \% \mathrm{Cl} 16.7$ to $19.6 \mu \mathrm{mol} / \mathrm{l} ; \mathrm{P}=0.003)$ for patients with a low vitamin B-12 concentration and 14.9 $\mu \mathrm{mol} / \mathrm{l}$ ( $95 \% \mathrm{Cl} 14.3$ to $15.5 \mu \mathrm{mol} / \mathrm{l}$; P 0.001 compared with vitamin $\mathrm{B}-12$ deficiency; $\mathrm{P}=0.005$ compared with low vitamin B-12) for patients with a normal vitamin B-12 concentration (>220 pmol/l).

Conclusions Long term treatment with metformin increases the risk of vitamin B-12 deficiency, which results in raised homocysteine concentrations. Vitamin B-12 deficiency is preventable; therefore, our findings suggest that regular measurement of vitamin B-12 concentrations during long term metformin treatment should be strongly considered.

Trial registration Clinicaltrials.gov NCT00375388.

\section{INTRODUCTION}

Metformin is considered a cornerstone in the treatment of diabetes and is the most frequently prescribed first line therapy for individuals with type 2 diabetes. ${ }^{1}$ In addition, it is one of a few antihyperglycaemic agents associated with improvements in cardiovascular morbidity and mortality, ${ }^{23}$ which is a major cause of death in patients with type 2 diabetes. ${ }^{4}$

There are few disadvantages to the use of metformin. Metformin does, however, induce vitamin B-12 malabsorption, which may increase the risk of developing vitamin B-12 deficiency ${ }^{5-7}$ - a clinically important and treatable condition. In addition, metformin treatment has been reported to be associated with decreased folate concentration, although the mechanism of this effect has not been elucidated. ${ }^{8}$ Finally, decreases in both folate and vitamin B-12 concentrations might, in turn, result in an increase in homocysteine concentrations (web figure A), an independent risk factor for cardiovascular disease, especially among individuals with type 2 diabetes. ${ }^{9-11}$

All current evidence on vitamin B-12 deficiency in metformin treatment comes from short term studies. ${ }^{5-712-14}$ No long term, placebo controlled data on the effects of metformin on concentrations of vitamin B-12 in patients with type 2 diabetes have been reported. In addition, placebo controlled data on the effects of metformin on homocysteine concentrations in type 2 diabetes are sparse, ${ }^{12}{ }^{15}$ and again no long term data are available. 


Enre-randomisation 12 weeks Short term treatment phase

Fig 1| HOME trial schedule

\section{Laboratory investigations}

Blood samples for this study were drawn at baseline and after 4, 17, 30, 43, and 52 months, and stored at $-80^{\circ} \mathrm{C}$ until analysis. Concentrations of vitamin B-12, folate, and homocysteine were measured in serum. Vitamin B-12 and folate concentrations were determined by an electrochemiluminescence immunoassay (ECLIA) using the competition principle. The mean intra-assay coefficients of variation for vitamin B-12 and folate were $2.3 \%$ and $3.5 \%$, respectively. The mean inter-assay coefficients were $2.9 \%$ and $4.7 \%$, respectively.

Total homocysteine concentration was measured using a kit from Chromsystems (Martinsried, Germany). The results were corrected against two types of "consensus plasma samples" (SKML, Nijmegen, the Netherlands) that had concentrations of $13 \mu \mathrm{mol} /$ 1 and $55 \mu \mathrm{mol} / 1$. The correction factor found was 0.90 . The intra-assay coefficients of variation were $2.2 \%$ and $1.8 \%$ at $12.8 \mu \mathrm{mol} / \mathrm{l}$ and $72.2 \mu \mathrm{mol} / \mathrm{l}$, respectively. The inter-assay coefficients of variation were $6.1 \%$ and $5.2 \%$ at $9.8 \mu \mathrm{mol} / 1$ and $21.1 \mu \mathrm{mol} / 1$, respectively.

In the HOME trial, vitamin B-12, folate, and homocysteine concentrations had been measured previously in samples obtained at baseline and after 16 weeks of treatment. ${ }^{12}$ To investigate the stability of the assay procedures, we compared the previously obtained values with values obtained for the present investigation. The correlation between old and new measurements of vitamin B-12 was 0.58 for baseline measurements and 0.91 for measurements taken after 16 weeks; for folate these values were 0.90 and 0.83 , respectively, and for homocysteine 0.99 and 0.99 , respectively. The relatively low correlation for vitamin B-12 values obtained at baseline was caused by five cases for which a large discrepancy existed between old and new values; these cases were subsequently excluded from analyses involving vitamin B-12.

\section{Statistical analysis}

Sample size calculations were based on expected differences in the occurrence of disease related end points, as described previously. ${ }^{3}$ With the sample size obtained, however, a decrease in vitamin B-12 concentration of $5 \%$ in the metformin group compared with the placebo group according ANCOVA tests should be detectable at a two sided 95\% confidence level, with a power of 0.82 .

We $\log$ transformed data on vitamin B-12, folate, and homocysteine concentrations before analysis because their distribution was skewed. Data are given as geometric means with $95 \%$ confidence intervals. Given that $\log$ values are not directly interpretable, the antilogs are reported instead. These values are the geometric mean percentage change from baseline.

The end point of interest was the percentage change of each variable from baseline at $4,17,30,43$, and 52 months, which was calculated from baseline values and the summary mean. The differences between the metformin and the placebo group were tested by a central $t$ test on $\log$ transformed values. We also calculated 


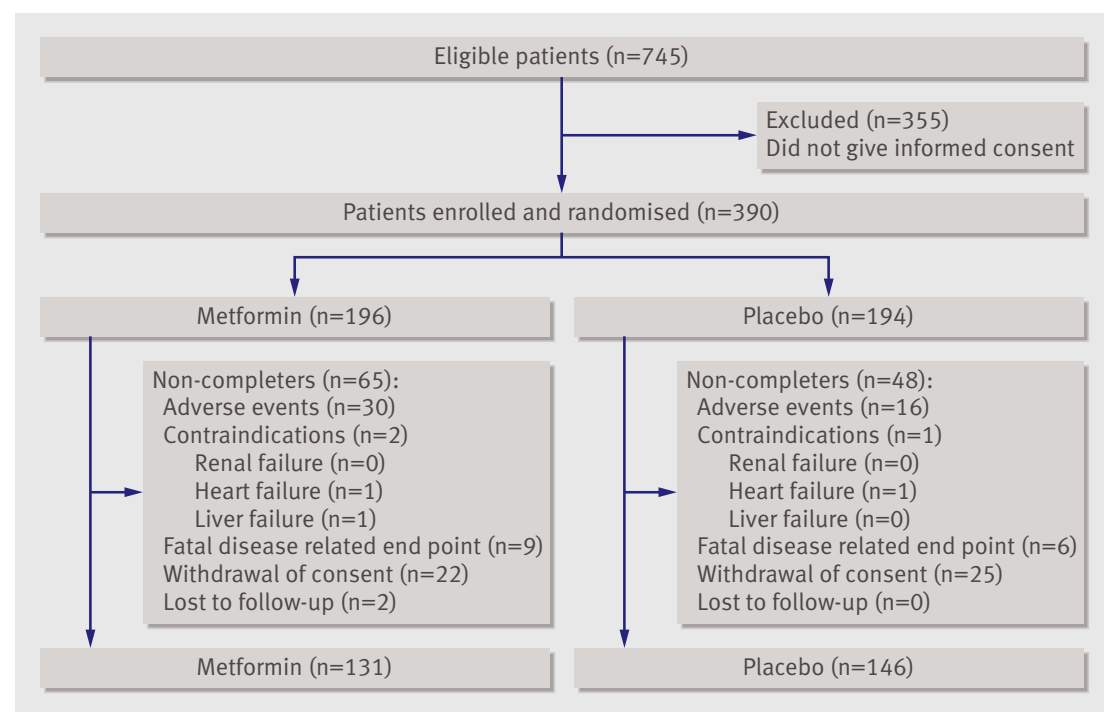

Fig 2| HOME patient profile more extensively elsewhere. ${ }^{3}$ Only two participants were lost to follow-up (at 33 and 26 months, respectively), both of whom were in the metformin group.

The actual mean dose in the metformin group was $2050 \mathrm{mg}$ a day. At the final visit, laboratory samples were available for 256 patients (127 metformin, 129 placebo). The main outcomes of this trial have been reported previously. ${ }^{3}$

Table 1 shows baseline characteristics of all patients analysed. Five randomised patients were excluded from the analysis because of poor correlations between old and newly measured vitamin B-12 values (see Methods). Patients randomised to metformin were older than those randomised to placebo ( $64 \pm 10$ years $v 59 \pm 11$ years), and were more likely to have a history of cardiovascular disease and less likely to be a smoker (30 (19\%) v $59(30 \%))$. The other characteristics were comparable between the two treatment groups.

Vitamin B-12, folate, and homocysteine concentrations During the 52 months of placebo treatment, vitamin B12 concentration increased from baseline by $0.2 \mathrm{pmol} /$ 1 ( $0 \%$ change, $95 \%$ confidence interval $-3 \%$ to $4 \%$ ), folate increased by $1.01 \mathrm{nmol} / 1(8 \%, 95 \%$ CI $4 \%$ to $12 \%$ ), and homocysteine increased by $1.60 \mu \mathrm{mol} / 1$ (20\%, 95\% CI 16\% to 25\%; fig 3). During metformin treatment, vitamin B-12 decreased by $89.8 \mathrm{pmol} / \mathrm{l}$ $(-19 \%, 95 \%$ CI $-22 \%$ to $-15 \%)$ from baseline, whereas folate concentration increased by $0.21 \mathrm{nmol} / 1$ (3\%, $95 \% \mathrm{CI}-1 \%$ to $6 \%$ ) and homocysteine concentration increased by $3.26 \mu \mathrm{mol} / 1(26 \%, 95 \%$ CI $21 \%$ to $31 \%)$.

Compared with placebo, metformin treatment was associated with a $19 \%$ decrease in vitamin B-12 concentration (95\% CI $-24 \%$ to $-14 \% ; \mathrm{P}<0.001)$ and a $5 \%$ decrease in folate concentration $(95 \% \mathrm{CI}-10 \%$ to $-0.4 \% ; \mathrm{P}=0.033)$, and a $5 \%$ increase in homocysteine concentrations $(95 \% \mathrm{CI}-1 \%$ to $11 \% ; \mathrm{P}=0.091)$. The effects of metformin on concentrations of vitamin B12 , folate, and homocysteine were re-analysed following adjustment for age, previous metformin treatment, duration of diabetes, gender, insulin dose, and smoking habits. None of these variables materially changed the results for vitamin B-12 and homocysteine (data not shown), but they did have an effect on the results for folate. After adjustment for body mass index and smoking, no significant effect of metformin on folate concentration was found (change in concentration compared with placebo $-0.1 \% ; \mathrm{P}=0.57)$.

At baseline, three patients $(1.6 \%)$ in the metformin group and four $(2.2 \%)$ in the placebo group had vitamin B-12 deficiency (vitamin B-12 concentration $<150 \mathrm{pmol} / \mathrm{l})$, whereas 14 patients $(7.3 \%)$ and 14 patients $(7.5 \%)$, respectively, had a low vitamin B-12 concentration (150-220 pmol/l). At the end of the study period, 19 patients $(9.9 \%)$ in the metformin group and five $(2.7 \%)$ in the placebo group had vitamin B-12 deficiency, whereas 35 patients $(18.2 \%)$ and 13 patients $(7.0 \%)$, respectively, had a low vitamin B-12 concentration.

The risk for vitamin B-12 deficiency at study end was 7.2 percentage points higher in the metformin group 


\begin{tabular}{|c|c|c|}
\hline & Metformin $(n=194)$ & Placebo $(n=191)$ \\
\hline \multicolumn{3}{|l|}{ Demographics } \\
\hline Men:women $(\mathrm{n}: \mathrm{n})$ & $81: 113$ & 95:96 \\
\hline Age (years) & $64(10)$ & 59 (11) \\
\hline Currently smoking (n (\%)) & 30 (19) & $59(30)$ \\
\hline Duration of diabetes (years) & $14(9)$ & $12(8)$ \\
\hline Insulin treatment (years) & 7 (8) & $6(6)$ \\
\hline \multicolumn{3}{|l|}{ Concomitant medication } \\
\hline Lipid lowering drugs (n (\%)) & $32(17)$ & $29(15)$ \\
\hline Blood pressure lowering drugs (n (\%)) & $91(47)$ & 74 (39) \\
\hline \multicolumn{3}{|l|}{ Metabolic variables } \\
\hline Weight (kg) & $85(16)$ & $87(15)$ \\
\hline Body mass index $\left(\mathrm{kg} / \mathrm{m}^{2}\right)$ & $30(5)$ & $30(5)$ \\
\hline \multicolumn{3}{|l|}{ Waist to hip ratio } \\
\hline Men & 1.02 & 1.02 \\
\hline Women & 0.93 & 0.92 \\
\hline Plasma glycated haemoglobin $\left(\mathrm{HbA}_{1}\right)$ & $7.9(1.2)$ & $7.9(1.2)$ \\
\hline Daily dose of insulin (IU/day) & 61 & 64 \\
\hline Systolic blood pressure $(\mathrm{mm} \mathrm{Hg})$ & $160(25)$ & $160(25)$ \\
\hline Diastolic blood pressure $(\mathrm{mm} \mathrm{Hg})$ & $86(12)$ & $86(11)$ \\
\hline Total cholesterol $(\mathrm{mmol} / \mathrm{l})$ & $5.6(1.3)$ & $5.5(1.2)$ \\
\hline Low density lipoprotein cholesterol (mmol/l) & $3.6(1.1)$ & $3.4(1.0)$ \\
\hline Triglycerides (mmol/l) & $1.7(1.2)$ & $1.9(1.5)$ \\
\hline High density lipoprotein cholesterol (mmol/l) & $1.3(0.4)$ & $1.3(0.4)$ \\
\hline Vitamin B-12 (pmol/l) & $378(130)$ & $380(135)$ \\
\hline Folate $(\mathrm{nmol} / \mathrm{l})$ & $18.7(7.2)$ & $18.7(7.5)$ \\
\hline Homocysteine $(\mu \mathrm{mol} / \mathrm{l})$ & $14.4(9.7)$ & $14.6(10.3)$ \\
\hline \multicolumn{3}{|l|}{ Previous cardiovascular disease } \\
\hline Myocardial infarction (n (\%)) & $24(12)$ & $21(11)$ \\
\hline Cardiovascular intervention (n (\%)) & $27(14)$ & $17(9)$ \\
\hline Stroke $(\mathrm{n}(\%))$ & $8(4)$ & $8(4)$ \\
\hline Non-traumatic amputation (n (\%)) & $5(3)$ & $3(2)$ \\
\hline
\end{tabular}

Values are mean (standard deviation) unless otherwise stated.

\section{Linear mixed model}

The interaction between treatment and time was a significant determinant of vitamin B-12 concentration $(\mathrm{P}=0.023)$ - that is, the lowering effect of metformin on vitamin B-12 concentrations increased with time. Body mass index and smoking were strong inverse determinants of folate concentration $(\mathrm{P}=0.003$ and $\mathrm{P}<0.0001$, respectively). There was no relation between time and folate concentration. After adjustment for body mass index and smoking, treatment with metformin was not a significant determinant of folate concentration, nor was the interaction between treatment and time $(\mathrm{P}=0.57$ and $\mathrm{P}=0.23$, respectively). Vitamin B-12 and folate levels were strong determinants of homocysteine concentration $(\mathrm{P}<0.0001)$. Homocysteine concentration increased with age at baseline $(\mathrm{P}<0.0001)$. There was no significant interaction between treatment and time for homocysteine concentrations $(\mathrm{P}=0.16)$.

\section{Additional analysis}

Per protocol analysis using only available data for those patients who remained in the trial until the final visit $(n=256)$ yielded similar results to our original intention to treat analysis (data not shown). General mixed model analysis yielded similar results to analysis using last observation carried forward (data not shown).

\section{DISCUSSION}

Our study on the long term effects of metformin treatment on serum concentrations of vitamin B-12, folate, and homocysteine in patients with type 2 diabetes treated with insulin had three main findings. Firstly, metformin significantly reduced concentrations of vitamin B-12, in accordance with findings from previous studies. ${ }^{131819}$ Importantly, our study shows that this decrease is not a transitory phenomenon, but persists and grows over time. Secondly, a small, significant decrease in folate concentrations was found in the metformin group compared with the placebo group; however, this reduction was not statistically significant after adjustments for body mass index and smoking. Thirdly, the decrease in vitamin B-12 concentrations was associated with an increase in homocysteine levels, which was not statistically significant. Further analyses, however, showed that homocysteine concentrations did increase in individuals in whom vitamin B-12 levels decreased below the concentration generally considered to indicate clinical deficiency - that is, $150 \mathrm{pmol} / \mathrm{l}$.

The finding of decreases in vitamin B-12 concentration during metformin treatment is not novel and has been reported before. A novel finding here, however, is that the decrease in vitamin B-12 levels is progressive. Furthermore, concentrations in some patients drop to the level at which most authorities agree vitamin substitution is required. This is also a novel finding, because although earlier trials in well fed, middle aged patients showed that metformin decreases vitamin B-12 concentrations, levels recorded remained within the normal range. ${ }^{5614}$ 
Metformin is thought to induce malabsorption of vitamin B-12 and intrinsic factor in the ileum, an effect that can be reversed by increasing calcium intake. ${ }^{618}$ The consequences of clinically important decreases in vitamin B-12 concentrations - such as macrocytic anaemia, neuropathy, and mental changes - can be profound. We note that there is no consensus on the issue of whether "asymptomatic" vitamin B-12 deficiency should be treated. ${ }^{20}$ However, studies show that some symptoms of vitamin B-12 deficiency are difficult to diagnose and can be irreversible, and treatment of vitamin B-12 deficiency is relatively easy, cheap, safe, and effective, ${ }^{21-24}$ in effect arguing in favour of treatment. In addition, although the necessity of treating "spontaneous" vitamin B-12 deficiency may be debated, one should be more easily inclined to treat drug induced vitamin B-12 deficiency, as a key principle of drug prescription is to do no harm. On the other hand, our study shows that it is reasonable to assume harm will eventually occur in some patients with metformin induced low concentrations of vitamin B-12.

Folate concentration increased in both the metformin group and the placebo group, possibly as a result of dietary counselling received by all patients throughout the trial. Our short term interim analysis showed a significantly larger increase in folate concentration in the placebo group, ${ }^{12}$ a finding that was initially replicated in the current analysis but that disappeared after adjustment for body mass index and smoking.

Previous studies have shown either no or small effects of metformin treatment on concentrations of homocysteine. ${ }^{13142526}$ We clearly show that homocysteine concentrations do increase with decreasing levels of vitamin B-12 (fig 4). The finding that metformin treatment significantly lowered concentrations of vitamin B-12 but did not significantly alter levels of homocysteine probably reflects the relatively low incidence of vitamin B-12 deficiency in the entire study population. As treatment with metformin continues, however, we expect that vitamin B-12 levels will continue to decrease, making increases in homocysteine concentrations inevitable in time.

\section{Strengths and limitations of study}

Strengths of our study include the randomised, placebo controlled, double blind design and its relatively long follow-up of 4.3 years, as well as frequent serum collection. Furthermore, the study was conducted in a non-academic setting and, therefore, the findings have high value in a community setting.

Another strength is that we used last observation carried forward in this analysis because this method is considered more conservative than general mixed model analysis, "freezing" any observed divergence between two groups by retaining the last observation made. In a mixed model analysis with missing data, estimations of future observations are made on the basis of observations made earlier in the trial, thereby reflecting a divergence more accurately but less conservatively.

Limitations of our study include the fact that we measured only total vitamin B-12 levels and not levels of

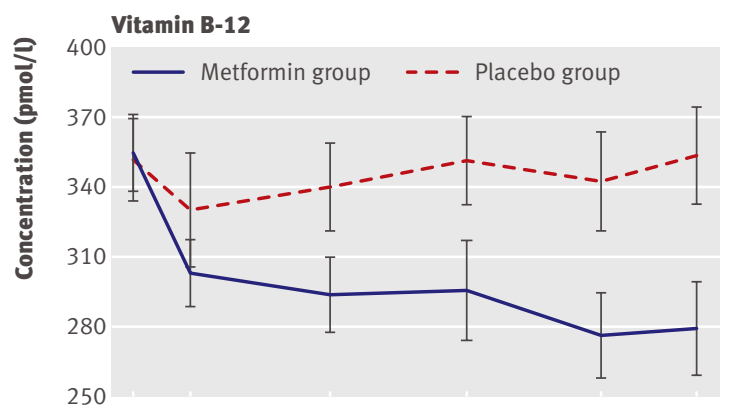

Number of available samples

Metformin group

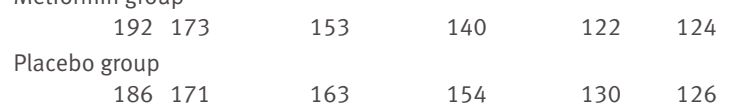

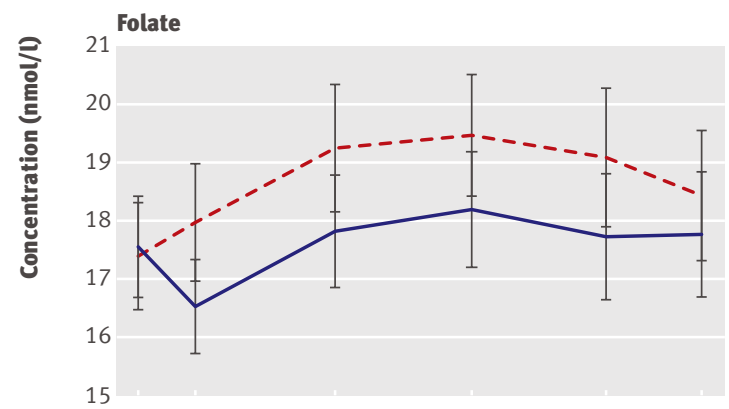

Number of available samples

Metformin group

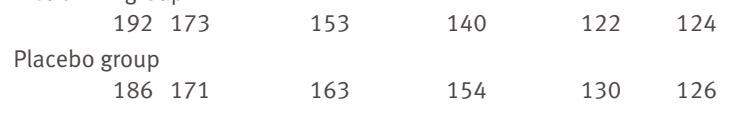

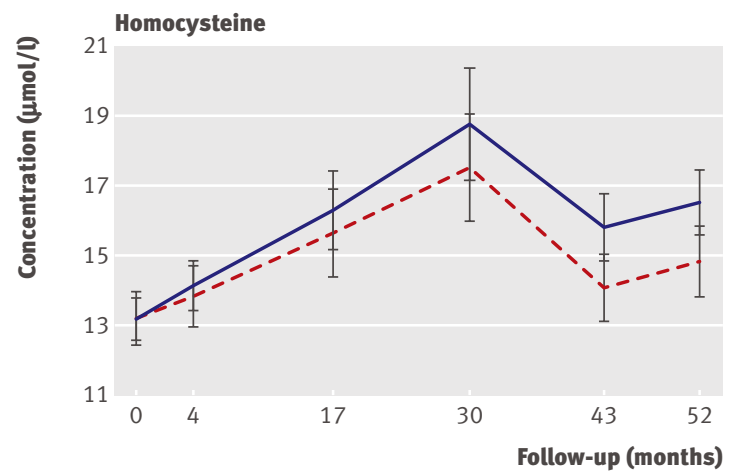

Number of available samples

Metformin group

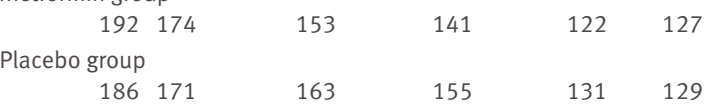

Fig 3 | Concentrations of vitamin B-12, folate, and homocysteine with $95 \%$ confidence intervals. Solid lines represent the metformin group, dotted lines the placebo group. Number of available samples for the metformin and placebo group is indicated. Five patients were excluded from the analysis because of poor correlations between old and newly measured vitamin B-12 values (see Methods). In addition, some patients had vitamin B-12 levels below 150 $\mathrm{pmol} / \mathrm{l}$ at baseline, at interim analysis, or at both stages, and were supplemented; these individuals were also excluded. 


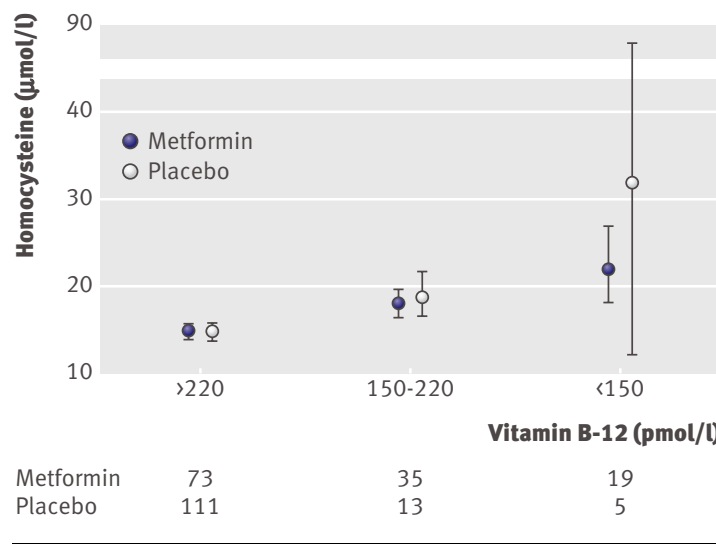

Fig 4 | Homocysteine concentrations with $95 \%$ confidence intervals for patients with a normal vitamin B-12 concentration ( $\$ 220 \mathrm{pmol} / \mathrm{l})$, a low vitamin B-12 concentration (150-220 pmol/l), and vitamin B-12 deficiency ( $(150 \mathrm{pmol} / \mathrm{l})$ after 4.3 years. The number of patients in each treatment group is indicated

holotranscobalamin II or methylmalonic acid, which may have been more precise indicators of vitamin B-12 status. Finally, it is likely that, if anything, we underestimated the impact of metformin treatment on the risk of clinically important vitamin B-12 deficiency. We showed that metformin treatment was associated not only with a raised risk of developing vitamin B-12 concentrations below $150 \mathrm{pmol} / \mathrm{l}$ but also with an elevated risk of developing vitamin B-12 levels between 150 and $220 \mathrm{pmol} / \mathrm{l}$, which is likely to represent clinically important vitamin B-12 deficiency in at least some individuals. ${ }^{27} \mathrm{~A}$ further reason that we may have somewhat underestimated the adverse effects of metformin is that all participants in our trial received frequent dietary counselling, which may have attenuated the impact of metformin treatment on vitamin status and may not be available in routine clinical practice.

\section{Conclusions and policy implications}

In conclusion, we showed that in patients with type 2 diabetes being treated with insulin, those additionally

\section{WHAT IS ALREADY KNOWN ON THIS TOPIC}

Metformin is considered a cornerstone in the treatment of type 2 diabetes and is frequently prescribed

Metformin is known to induce malabsorption of vitamin B-12 and may be associated with decreased folate concentrations, which might, in turn, result in an increase in homocysteine concentrations

Few and only short term data exist on the effect of metformin treatment on vitamin B-12, folate, and homocysteine

\section{WHAT THIS STUDY ADDS}

Long term treatment with metformin in patients with type 2 diabetes receiving insulin increases the risk of vitamin B-12 deficiency, which results in higher levels of homocysteine

The negative effect of metformin on vitamin B-12 concentrations increases over time

Our data provide a strong case for routine assessment of vitamin B-12 levels during long term treatment with metformin treated with metformin had a seven percentage point greater absolute risk of vitamin B-12 deficiency than those treated with placebo during 4.3 years of followup. In addition, the reduction in vitamin B-12 concentration associated with metformin increased with time.

Current guidelines indicate that metformin is a cornerstone in the treatment of type 2 diabetes, but make no recommendations on the detection and prevention of vitamin B-12 deficiency during treatment. Our data provide a strong case for routine assessment of vitamin B-12 levels during long term treatment with metformin.

Contributors: AK, AJMD, and CDAS are responsible for the study concept and design. JdJ, AK, MGW, and DB collected the data, and statistical analysis was conducted by PL and JdJ. JdJ, AK, PL, and CDAS undertook analysis and interpretation of the data. JdJ, AK, and CDAS drafted the manuscript, and AK, AJMD, and CDAS undertook critical revisions of the manuscript for important intellectual content. Administrative, technical or material support was provided by JvdK, DB, JV, MGW, JdJ, and AK. JdJ, AK and PL had full access to all of the data in the study and take responsibility for the integrity of the data and the accuracy of the data analysis. Jd, AK, and CDAS accept full responsibility for the work and the conduct of the study, had access to the data, and controlled the decision to publish, and as such act as guarantors for the study.

Funding: Hyperinsulinaemia: the Outcome of its Metabolic Effects (HOME) trial was supported by grants from Altana, Lifescan, Merck Santé, Merck Sharp \& Dohme, and Novo Nordisk. The sponsors had no role in the design and conduct of the study; in the collection, analysis, and interpretation of the data; or in the preparation, review, or approval of the manuscript.

Competing interests: All authors have completed the Unified Competing Interest form at www.icmje.org/coi_disclosure.pdf (available on request from the corresponding author) and all authors want to declare: (1) Financial support for the submitted work from Merck Sharp \& Dohme. All authors also declare: (2) No financial relationships with commercial entities that might have an interest in the submitted work; (3) No spouses, partners, or children with relationships with commercial entities that might have an interest in the submitted work; and (4) No nonfinancial interests that may be relevant to the submitted work. Ethical approval: The medical ethical committees of the three participating hospitals approved the trial protocol. The trial has been conducted in accordance with the Committee for Medicinal Products for Human Use note for guidance on good clinical practice (CPMP/ICH/135/ 95), dated 17 July 1996, and in accordance with the Declaration of Helsink (revised version of Hong Kong in 1989 and Edinburgh in 2000). All patients gave written informed consent.

Data sharing: No additional data available.

1 Kirpichnikov D, McFarlane SI, Sowers JR. Metformin: an update. Ann Intern Med 2002;137:25-33.

2 UK Prospective Diabetes Study (UKPDS) Group. Effect of intensive blood-glucose control with metformin on complications in overweight patients with type 2 diabetes (UKPDS 34). Lancet 1998;352:854-65.

3 Kooy A, de Jager J, Lehert P, Bets D, Wulffele MG, Donker AJM, et al. Long-term effects of metformin on metabolism and microvascular and macrovascular disease in patients with type 2 diabetes mellitus. Arch Intern Med 2009;169:616-25.

4 Pyorala K, Laakso M, Uusitupa M. Diabetes and atherosclerosis: an epidemiologic view. Diabetes Metab Rev 1987;3:463-524.

5 DeFronzo RA, Goodman AM, the Multicenter Metformin Study Group. Efficacy of metformin in patients with non-insulin-dependent diabetes mellitus. N Engl J Med 1995;333:541-9.

6 Bauman WA, Shaw S, Jayatilleke E, Spungen AM, Herbert V. Increased intake of calcium reverses vitamin B12 malabsorption induced by metformin. Diabetes Care 2000;23:1227-31.

7 Ting RZ-W, Szeto CC, Chan MH-M, Ma KK, Chow KM. Risk factors of vitamin B12 deficiency in patients receiving metformin. Arch Intern Med 2006;166:1975-9.

8 Carlsen SM, Folling I, Grill V, Bjerve KS, Schneede J, Refsum H. Metformin increases total serum homocysteine levels in nondiabetic male patients with coronary heart disease. Scand J Clin Lab Invest 1997;57:521-7.

9 Boushey CJ, Beresford SA, Omenn GS, Motulsky AG. A quantitative assessment of plasma homocysteine as a risk factor for vascular 
disease. Probable benefits of increasing folic acid intakes. JAMA 1995;274:1049-57.

10 Nygård O, Nordrehaug JE, Refsum H, Ueland PM, Farstad M, Vollset SE. Plasma homocysteine levels and mortality in patients with coronary artery disease. N Engl J Med 1997;337:230-6.

11 Welch GN, Loscalzo J. Homocysteine and atherothrombosis. N Engl J Med 1998;338:1042-50.

12 Wulffelé MG, Kooy A, Lehert P, Bets D, Ogterop JC, Borger van der Burg B, et al. Effects of short-term treatment with metformin on serum concentrations of homocysteine, folate, and vitamin B12 in type 2 diabetes mellitus: a randomized, placebo-controlled trial. J Intern Med 2003;254:455-63.

13 Pongchaidecha M, Srikusalanukul V, Chattananon A, Tanjariyaporn S. Effect of metformin on plasma homocysteine, vitamin B12 and folic acid: a cross-sectional study in patients with type 2 diabetes mellitus. J Med Assoc Thai 2004;87:780-7.

14 Sahin M, Tutuncu NB, Ertugrul D, Tanaci N, Guvener ND. Effects of metformin or rosiglitazone on serum concentrations of homocysteine, folate, and vitamin B12 in patients with type 2 diabetes mellitus. J Diabetes Complications 2007;21:118-23.

15 Hermann LS, Kalén J, Katzman P, Lager I, Nilsson A, Norrhamn O, et al. Long-term glycaemic improvement after addition of metformin to insulin in insulin-treated obese type 2 diabetes patients. Diabetes Obes Metab 2001;3:428-34.

16 Wulffelé MG, Kooy A, Lehert P, Bets D, Ogterop JC, Borger van der Burg B, et al. Combination of insulin and metformin in the treatment of type 2 diabetes. Diabetes Care 2002;25:2133-40.

17 Marks PW, Zuckerberg LR. Case 30-2004: a 37-year-old woman with paresthesias of the arms and legs. N Engl J Med 2004;351:1333-41.
18 Adams JF, Clark JS, Ireland JT, Kesson CM, Watson WS. Malabsorption of vitamin B12 and intrinsic factor secretion during biguanide therapy. Diabetologia 1983;24:16-8.

19 Tomkin GH, Hadden DR, Weaver JA, Montgomery DA. Vitamin-B12 status of patients on long-term metformin therapy. BMJ 1971;2:685-7.

20 British Committee for Standards in Haematology guidelines. http:// www.bcshguidelines.com/.

21 Healton EB, Savage DG, Brust JC, Garrett TJ, Lindenbaum J. Neurologic aspects of cobalamin deficiency. Medicine 1991;70:229-45.

22 Stabler SP, Allen RH, Savage DG, Lindenbaum J. Clinical spectrum and diagnosis of cobalamin deficiency. Blood 1990;76:871-81.

23 Kuzminski AM, Giacco AJD, Allen RH, Stabler SP, Lindenbaum J. Effective treatment of cobalamin deficiency with oral cobalamin. Blood 1998;92:1191-8.

24 Hermann W, Obeid R. Causes and early diagnosis of vitamin B12 deficiency. Dtsch Arztebl Int 2008;105:680-5.

25 Derosa G, Mugellini A, Ciccarelli L, Crescenzi G, Fogari R. Comparison of glycaemic control and cardiovascular risk profile in patients with type 2 diabetes during treatment with either repaglinide or metformin. Diabetes Res Clin Pract 2003;60:161-9.

26 Hoogeveen EK, Kostense PJ, Jakobs C, Bouter LM, Heine RJ, Stehouwer CDA. Does metformin increase the serum total homocysteine level in non-insulin-dependent diabetes mellitus? J Intern Med 1997;242:389-94.

27 Snow CF. Laboratory diagnosis of vitamin B12 and folate deficiency. Arch Intern Med 1999;159:1289-98.

Accepted: 25 February 2010 